\title{
LC-MS-based absolute metabolite quantification: application to metabolic flux measurement in trypanosomes
}

\author{
Dong-Hyun Kim ${ }^{1,2} \cdot$ Fiona Achcar $^{1} \cdot$ Rainer Breitling $^{3} \cdot$ Karl E. Burgess $^{4}$. \\ Michael P. Barrett ${ }^{1,4}$
}

Received: 27 March 2015/Accepted: 25 June 2015/Published online: 9 July 2015

(c) The Author(s) 2015. This article is published with open access at Springerlink.com

\begin{abstract}
Human African trypanosomiasis is a neglected tropical disease caused by the protozoan parasite, Trypanosoma brucei. In the mammalian bloodstream, the trypanosome's metabolism differs significantly from that of its host. For example, the parasite relies exclusively on glycolysis for energy source. Recently, computational and mathematical models of trypanosome metabolism have been generated to assist in understanding the parasite metabolism with the aim of facilitating drug development. Optimisation of these models requires quantitative information, including metabolite concentrations and/or metabolic fluxes that have been hitherto unavailable on a large scale. Here, we have implemented an LC-MS-based method that allows large scale quantification of metabolite levels by using $\mathrm{U}_{-}{ }^{13} \mathrm{C}$-labelled $E$. coli extracts as internal
\end{abstract}

Electronic supplementary material The online version of this article (doi:10.1007/s11306-015-0827-2) contains supplementary material, which is available to authorized users.

Michael P. Barrett

michael.barrett@glasgow.ac.uk

1 Wellcome Trust Centre for Molecular Parasitology, Institute of Infection, Immunity and Inflammation, College of Medical Veterinary and Life Sciences, University of Glasgow, Glasgow G12 8TA, UK

2 Present Address: Centre for Analytical Bioscience, School of Pharmacy, University of Nottingham, University Park, Nottingham NG7 2RD, UK

3 Manchester Centre of Synthetic Biology for Fine and Speciality Chemicals, Manchester Institute of Biotechnology, Faculty of Life Sciences, University of Manchester, Manchester M1 7DN, UK

4 Glasgow Polyomics, Wolfson Wohl Cancer Research Centre, College of Medical Veterinary \& Life Sciences, University of Glasgow, Glasgow G61 1QH, UK standards. Known amounts of labelled E. coli extract were added into the parasite samples, as well as calibration standards, and used to obtain calibration curves enabling us to convert intensities into concentrations. This method allowed us to reliably quantify the changes of 43 intracellular metabolites and 32 extracellular metabolites in the medium over time. Based on the absolute quantification, we were able to compute consumption and production fluxes. These quantitative data can now be used to optimise computational models of parasite metabolism.

Keywords Trypanosome $\cdot$ LC-MS $\cdot$ Absolute metabolite quantification - Metabolic flux $\cdot{ }^{13} \mathrm{C}$-labelled E. coli

\section{Introduction}

Human African trypanosomiasis (HAT), also known as sleeping sickness, is a neglected tropical disease in subSaharan Africa caused by the protozoan parasite Trypanosoma brucei and transmitted by tsetse flies (Barrett et al. 2007; Brun et al. 2010). HAT is fatal if untreated, but currently used trypanocidal drugs are difficult to administer, can cause severe side effects and suffer from emerging parasite resistance (Delespaux and de Koning 2007; Vincent et al. 2012). Therefore, new drugs are urgently required to treat the disease, and improved knowledge of the metabolism of the parasite is necessary to facilitate drug development. Computational models can be used to guide our analysis of parasite physiology, and they have been used in understanding T. brucei metabolism, where it is hoped that they will play a critical role in developing optimised anti-parasite drugs (Bakker et al. 2010). However, such models require quantitative measurements, including data on concentrations and fluxes of metabolites, 
for their optimisation. Such data have so far been collected on a small scale, targeting individual reactions and metabolites (Achcar et al. 2013, 2012; Albert et al. 2005; Bakker et al. 2000, 1997; Kerkhoven et al. 2013), but a full understanding of parasite physiology would benefit from a more global quantitative assessment of metabolite dynamics.

LC-MS-based metabolomic studies enable simultaneous measurement of multiple small molecules in a biological system (Theodoridis et al. 2012; Vincent et al. 2012). The resulting data have been widely used to investigate the relative changes in intracellular metabolite concentrations for biomarker discovery (Denery et al. 2010; van der Kloet et al. 2012). However, a full understanding of cellular responses requires absolute metabolite concentrations, which can feed into quantitative computational models of metabolism.

LC-ESI/MS is a powerful analytical tool for quantification and identification of small molecules (metabolites) by providing retention time and exact molecular weights. However, it has some disadvantages, including nonlinear responses due to detector response and matrix effects (Annesley 2003). This drawback can lead to nonlinear calibration curves for quantification, so that true levels of intracellular metabolites are difficult to ascertain (Shi 2003). In addition, variations in instrumental response and degradation of metabolites of interest during sample preparation can also result in a biased quantitative result (Vuckovic 2012). In order to overcome these challenges, uniformly (U)- ${ }^{13} \mathrm{C}$-labelled isotopomers of metabolites can be added as internal standards, behaving identically to their unlabelled equivalents in sample extracts. The demonstrated fidelity of Orbitrap mass spectrometers for quantifying isotope ratios (Xu et al. 2010) makes them particularly suitable for this purpose. However, since the availability of commercial $\mathrm{U}^{13} \mathrm{C}$-labelled isotopomers is limited and they are often prohibitively expensive, in vivo synthesis of $\mathrm{U}_{-}{ }^{13} \mathrm{C}$-labelled compounds is required using suitable microorganisms grown in $\mathrm{U}^{-13} \mathrm{C}$-labelled glucoselimited culture media. Absolute intracellular concentrations of metabolites in a sample can then be calculated by adding known amounts of $\mathrm{U}^{-13} \mathrm{C}$-labelled cell extract prior to the extraction procedure (Bennett et al. 2009; Mashego et al. 2004). The most suitable method for this isotope ratiobased MS (IR-MS) technique is to use the same organism for generating U- ${ }^{13} \mathrm{C}$-labelled cell extracts which can cover all of intracellular metabolites for absolute quantification (Mashego et al. 2004; Wu et al. 2005). However, T. brucei cannot be used for this purpose, as media containing only glucose as carbon source are not available (Creek et al. 2013); hence, it is not possible to obtain all metabolites present in the cell in fully labelled form. Furthermore, $T$. brucei reaches a relatively low cell density when cultivated. Consequently, we decided to explore the utility of $\mathrm{U}^{13} \mathrm{C}$-labelled $E$. coli extract as an alternative source of isotope analogues for quantitative analysis of trypanosome metabolites.

In this paper, we report the first application of the IRMS technique using $\mathrm{U}_{-}{ }^{13} \mathrm{C}$-labelled $E$. coli extracts for the global quantitative analysis of intra- and extra-cellular metabolite concentrations in trypanosomes. We analyse the overlap of E. coli and T. brucei metabolomes to demonstrate the viability of the approach. Furthermore, by measuring absolute concentrations of extracellular metabolites in spent media, we were able to compute metabolic fluxes.

\section{Materials and methods}

\subsection{Preparation of $\mathrm{U}-{ }^{13} \mathrm{C}$-labelled $E$. coli extract and calibration standards}

E. coli was cultured on $100 \%$ U- ${ }^{13} \mathrm{C}$-labelled glucose and the labelled metabolites were extracted using existing sampling procedures (Rabinowitz and Kimball 2007; Winder et al. 2008). Briefly, E. coli MG1655 was grown in $500 \mathrm{ml}$ of M9 minimal medium which contained the following per litre: $100 \mathrm{ml}$ of $5 \times \mathrm{M} 9$ minimal medium (33.9 $\mathrm{g}$ of $\mathrm{Na}_{2} \mathrm{HOP}_{4}, 15 \mathrm{~g}$ of $\mathrm{KH}_{2} \mathrm{PO}_{4}, 2.5 \mathrm{~g}$ of $\mathrm{NaCl}$ ), $1 \mathrm{ml}$ of $1 \mathrm{M} \mathrm{MgSO}_{4}, 1 \mathrm{ml}$ of $1 \mathrm{M} \mathrm{CaCl}_{2}, 3 \mathrm{ml}$ of $2 \mathrm{M}$ $\left(\mathrm{NH}_{4}\right)_{2} \mathrm{SO}_{4}$ and $5 \mathrm{ml}$ of $20 \%$ U- ${ }^{13} \mathrm{C}$-labelled glucose at $37{ }^{\circ} \mathrm{C}$ overnight. The culture was grown up to an optical density at $600 \mathrm{~nm}\left(\mathrm{OD}_{600}\right)$ of $\sim 1.0$ and the cells were centrifuged at $3000 \times g$ for $10 \mathrm{~min}$. The supernatant was removed and $8 \mathrm{ml}$ of $-48{ }^{\circ} \mathrm{C}$ acetonitrile/methanol/water $(2: 2: 1)$ was added into the cell pellet for quenching metabolism and extracting metabolites. Then, cells were freezethaw extracted (i.e., flash frozen in liquid $\mathrm{N}_{2}$ for $1 \mathrm{~min}$ and thawed at $4{ }^{\circ} \mathrm{C}$, then vortexed for $30 \mathrm{~s} \times 4$ ) and centrifuged at $3000 \times g$ for $10 \mathrm{~min}$. The supernatant was collected and stored at $-80{ }^{\circ} \mathrm{C}$ prior to analysis. The $\mathrm{U}-{ }^{13} \mathrm{C}$ labelled extract provides a range of $\mathrm{U}_{-}{ }^{13} \mathrm{C}$-labelled internal standards for each intracellular metabolite being investigated. Furthermore, absolute metabolite concentrations were obtained by adding the same amounts of the labelled $E$. coli extract to both cell samples prior to the extraction procedure and the unlabelled calibration standards.

The calibration standards for the absolute quantification of metabolites were prepared by diluting main stock solutions of unlabelled compounds obtaining seven concentration points $(0.001,0.01,0.1,1,10,50$ and $100 \mu \mathrm{M}$ or $0.03,0.3,3,30,300,600,1200$ and $3000 \mu \mathrm{M})$. The standard samples were split into five groups according to their masses (see Supplementary Table S2), so that metabolites of similar mass do not overlap, and a clear and single peak of each metabolite in the MS data can be provided for 
calibration curves. Then, the $\mathrm{U}_{-}{ }^{13} \mathrm{C}$-labelled $E$. coli extract was added to each calibration sample in a 1:4 ratio.

\subsection{Parasite growth conditions and metabolite extraction}

For intracellular concentrations, T. brucei wild type strain 427 bloodstream forms were cultured in HMI-9 (Hirumi et al. 1977) or Creek's minimal medium (CMM) (Creek et al. 2013) supplemented with $10 \%$ fetal bovine serum at $37{ }^{\circ} \mathrm{C}, 5 \% \mathrm{CO}_{2} 2 \times 10^{4}$ cells $/ \mathrm{ml}$ were seeded in $\mathrm{T} 150$ culture flasks and allow them to reach a density of $2 \times 10^{6}$ cells $/ \mathrm{ml}$. Appropriate volumes of cell culture were taken to yield $5 \times 10^{7}$ cells and quenched in EtOH/dry ice bath as previously described ( $t$ 'Kindt et al. 2010). Cells were centrifuged at $1250 \times \mathrm{g}$ for $10 \mathrm{~min}$ and then the growth medium was removed and the cells were washed with $1 \mathrm{ml}$ of PBS pre-cooled at $4{ }^{\circ} \mathrm{C}$. The cell solution was centrifuged again at $1250 \times \mathrm{g}$ for $5 \mathrm{~min}$ and the supernatant was removed. Metabolites were extracted by adding $100 \mu \mathrm{l}$ of chloroform/methanol/water (1:3:1) spiked with the $\mathrm{U}^{13} \mathrm{C}$-labelled E. coli extract in a $1: 4$ ratio and mixed vigorously for $1 \mathrm{~h}$ at $4{ }^{\circ} \mathrm{C}$. After centrifugation at $13,000 \times g$ for $10 \mathrm{~min}$, the supernatant was collected and stored at $-80{ }^{\circ} \mathrm{C}$ until LC-MS analysis. One pooled quality control (QC) sample was prepared by mixing all the samples in order to assess instrument performance. All samples were prepared in four biological replicates.

For extracellular concentrations, $0.38 \times 10^{4}$ cells $/ \mathrm{ml}$ were seeded in $40 \mathrm{ml}$ of CMM or HMI-9 and incubated at $37{ }^{\circ} \mathrm{C}, 5 \% \mathrm{CO}_{2}$ for $57 \mathrm{~h}$. The cells were sampled for analysis after $0,7,24,31,48,51$ and $57 \mathrm{~h}$. During the incubation period, $5 \mathrm{ml}$ of cell culture was taken and cells were counted. Then, the cell culture was centrifuged at $1250 \times g$ for $10 \mathrm{~min}$ and $10 \mu \mathrm{l}$ of supernatant (spent medium) was taken for extracellular concentration measurements. The harvested spent medium was extracted by adding $190 \mu \mathrm{l}$ of chloroform/methanol/water (1:3:1) spiked with the $\mathrm{U}^{13} \mathrm{C}$-labelled $E$. coli extract in a 1:4 ratio and mixed vigorously, then stored at $-80{ }^{\circ} \mathrm{C}$ prior to analysis. All samples were prepared in five biological replicates.

\subsection{Analytical methodologies}

Separations were performed on a Dionex UltiMate 3000 RSLC liquid chromatography system (Dionex, Camberley, Surrey). Chromatography was carried out using a ZIC-HILIC or ZIC-pHLIC $150 \times 4.6 \mathrm{~mm}^{2}, 5 \mu \mathrm{m}$ column (Merck Sequant) as previously described (Creek et al. 2011). Briefly, the column was maintained at $30{ }^{\circ} \mathrm{C}$ and samples were eluted with a linear gradient from $80 \% \mathrm{~B}$ to $20 \% \mathrm{~B}$ over $32 \mathrm{~min}$, followed by an 8 min wash with $5 \% \mathrm{~B}$, and 8 min re-equilibration with $80 \% \mathrm{~B}$ at the flow rate of $300 \mu \mathrm{l} / \mathrm{min}$. Mobile phase A was water $(0.1 \% \mathrm{v} / \mathrm{v}$ formic acid $)$ for a ZIC-HILIC or $20 \mathrm{mM}$ ammonium carbonate in water for a ZIC-pHILIC column and mobile phase B was acetonitrile $(0.08 \% \mathrm{v} / \mathrm{v}$ formic acid) for a ZIC-HILIC or $100 \%$ acetonitrile for a ZICpHILIC column. The injection volume was $10 \mu \mathrm{l}$ and samples were maintained at $4{ }^{\circ} \mathrm{C}$. For the MS analysis, an Orbitrap Exactive (Thermo Fisher Scientific, Hemel Hempstead, UK) with a HESI-II probe was operated in polarity switching mode, with the following settings as previously described (Creek et al. 2011): resolution 50,000, AGC $1 \times 10^{6}, \mathrm{~m} / \mathrm{z}$ range $70-1400$, sheath gas 40 , auxiliary gas 20 , sweep gas 1 , probe temperature $150^{\circ} \mathrm{C}$, and capillary temperature $275^{\circ} \mathrm{C}$. For positive mode ionisation: source voltage $+4 \mathrm{kV}$, capillary voltage $+50 \mathrm{~V}$, tube voltage $+70 \mathrm{kV}$, skimmer voltage $+20 \mathrm{~V}$. For negative mode ionisation: source voltage $-3.5 \mathrm{kV}$, capillary voltage $-50 \mathrm{~V}$, tube voltage $-70 \mathrm{~V}$, skimmer voltage $-20 \mathrm{~V}$. Mass calibration was performed for each polarity immediately before each analysis batch. The calibration mass range was extended to cover small metabolites by inclusion of low-mass contaminants with the standard Thermo calibration mixture masses (below $\mathrm{m} / \mathrm{z}$ 1400), $\mathrm{C}_{2} \mathrm{H}_{6} \mathrm{NO}_{2}$ for positive ion electrospray ionisation (PIESI) mode $(\mathrm{m} / \mathrm{z} 76.0393)$ and $\mathrm{C}_{3} \mathrm{H}_{5} \mathrm{O}_{3}$ for negative ion electrospray ionisation (NIESI) mode ( $\mathrm{m} / \mathrm{z}$ 89.0244). To enhance calibration stability, lock-mass correction was also applied to each analytical run using these ubiquitous low-mass contaminants.

\subsection{Data processing and analysis}

Raw LC-MS data were processed with XCMS for untargeted peak-picking (Tautenhahn et al. 2008) and mzMatch for peak matching and annotation of related peaks (Scheltema et al. 2011). Noise filtering and putative metabolite identification was performed by IDEOM using the default parameters (Creek et al. 2012). Metabolite identification was performed by matching accurate masses and retention times of authentic standards [Level 1 metabolite identification according to the metabolomics standards initiative (Sumner et al. 2014, 2007)], but when standards were not available, predicted retention times were employed, hence these identifications should be considered as putative (Level 2 identification).

For the extracellular medium quantifications, raw LCMS data were processed with mzMatch and mzMatch-ISO (Chokkathukalam et al. 2013) (see R script in Supplementary File A). The peaks matching accurate mass and retention time with our calibration standards were then selected for interpretation.

\subsection{Estimation of metabolic fluxes}

Among the 192 calibration standards examined, 32 had sufficiently clear single peaks in the $T$. brucei extracts, in 
the calibration standard and in the labelled $E$. coli extracts to allow absolute quantification. Calibration curves for these were constructed by calculating the ratio between the unlabelled $\mathrm{U}^{12} \mathrm{C}$ peaks (from the standards) and the $\mathrm{U}-{ }^{13} \mathrm{C}$-labelled peaks (from E. coli). Using these calibration curves and the $\mathrm{U}_{-}{ }^{12} \mathrm{C}$ over $\mathrm{U}_{-}{ }^{13} \mathrm{C}$ ratios calculated from the spent medium time course (cells grown in CMM media), absolute concentrations of these 32 metabolites were calculated (see Sect. 3). Absolute concentrations of eight metabolites were also quantified in the spent medium of cells grown in HMI-9 medium for comparison. From these time courses, production or consumption fluxes could be estimated, assuming that these fluxes are constant over time. Supposing exponential growth, Eq. 1 expresses the cell density as a function of time:

$c(t)=c_{0} \times \exp (g \times t)$,

where $c_{0}$ is the cell density at time $\mathrm{t}=0, g$ is the growth rate.

Assuming constant consumption/production flux, Eq. 2 describes the concentration of a metabolite $m$ as a function of time $t$ :

$m(t)=\left(k \times \frac{c_{0}}{g}\right) \times(\exp (g \times t)-1)+m_{0}$,

where $k$ is the flux and $m_{o}$ is the concentration of metabolite $m$ at time $\mathrm{t}=0$. Given Eqs. 1 and 2, the concentration of a metabolite $m$ as a function of cell density can be expressed by Eq. 3:

$$
\begin{gathered}
m(t)=a \times c(t)+b \\
a=\frac{k}{g}, b=-k \times \frac{c_{0}}{g}+m_{0}
\end{gathered}
$$

Parameters $g$ and $c_{0}$ were estimated by fitting the equation to the data using the python package lmfit (http://cars9. uchicago.edu/software/python/lmfit/) with the parameters $\mathrm{g}$ and $c_{0}$ bounded below at 0 . Similarly, $k$ and $m_{0}$ were estimated via $a$ and $b$ by fitting Eq. 3. The flux $k$ was then converted from $\mathrm{nmol} /(\mathrm{h}$ cell $)$ to $\mathrm{nmol} /\left(\min 10^{8}\right.$ cells $)$.

\section{Results and discussion}

\subsection{Comparison of the E. coli and T. brucei metabolomes}

The metabolomes of E. coli and T. brucei were investigated by an untargeted LC-ESI/MS technique to evaluate which metabolites can be quantified by the IR-MS technique. The observed chemical compositions of both species are shown in Fig. 1a, b.

Totals of 460 and 477 metabolites were putatively identified in E. coli and T. brucei extracts, respectively.
Detected intracellular metabolites were dominated by a few chemical classes in both species: metabolites predicted to be related to the metabolism of amino acids (E. coli, $32.2 \%$; T. brucei, $28.7 \%$ ), carbohydrates (E. coli, $10.2 \%$; T. brucei, $12.6 \%$ ), nucleotides (E. coli, $8.0 \%$; T. brucei, $7.5 \%$ ), and cofactors and vitamins (E. coli, $8.2 \% ; T$. brucei, $3.6 \%$ ). With the exception of compounds which were not assigned to any specific class of metabolites, amino acid metabolism-related metabolites were the most numerous compounds detected in the both extracts, followed by compounds involved in carbohydrate metabolism. Of the measured metabolites in the both extracts, 198 metabolites identified by the technique were common, as shown in Fig. 1c, and thus are potentially amenable to absolute quantification as described here. Many core metabolites, such as amino acids, carbohydrates and nucleotides, were cross-detected in both species, confirming our ability to investigate central metabolism of trypanosomes by measuring metabolic fluxes using the IR-MS technique with easily generated labelled $E$. coli extracts. The complete list of the common putative metabolites is available in Supplementary Table S3.

\subsection{Absolute quantitative analysis of intracellular metabolites in $T$. brucei using LC-ESI/MS}

An absolute quantitative analysis of intra- and extra-cellular metabolites in T. bruce $i$ was carried out. To determine intracellular concentrations of metabolites in the parasite, sets of calibration standard mixtures of known concentrations added with $\mathrm{U}^{13} \mathrm{C}$-labelled $E$. coli extract in a $1: 4$ ratio were analysed by LC-ESI/MS. Calibration curves of each metabolite containing a minimum of five concentration points were then obtained by comparison of the ratios of mass spectral area from unlabelled $(\mathrm{M}+0)$ and labelled $\{\mathrm{M}+(\mathrm{N} \times 1.0033)\}$ metabolites, where $\mathrm{M}$ is the monoisotopic mass $\left({ }^{12} \mathrm{C}-\right)$ and $\mathrm{N}$ is the number of carbon atoms of the molecule. Coefficients of determination showed very good linearity of the calibration curves of 43 intracellular metabolites $\left(\mathrm{R}^{2}>0.99\right.$, data not shown).

The absolute concentrations of 43 intracellular metabolites were determined against the calibration curves. The concentrations of intracellular metabolites are shown in Table 1. Of 43 measured intracellular metabolites, Lalanine was the most abundant compound $(21.4 \mathrm{mM}-$ HMI-9; $32.8 \mathrm{mM}-\mathrm{CMM}$ ) in the cell extract, followed by pyruvate $(7.2 \mathrm{mM}-\mathrm{HMI}-9 ; 7.1 \mathrm{mM}-\mathrm{CMM})$, L-proline (3.8 $\mathrm{mM}-\mathrm{HMI}-9 ; \quad 2.7 \mathrm{mM}-\mathrm{CMM}$ ) and spermidine (3.5 mM-HMI-9; $4.2 \mathrm{mM}-\mathrm{CMM}$ ). The total concentration of the less abundant metabolites $(50 \%$ of all metabolites measured) was only $0.6 \mathrm{mM}$.

For the validation of our LC-MS-based absolute quantification method, the measured intracellular concentrations 
Fig. 1 Comparison of the measured metabolomes extracted from E. coli (a) and $T$. brucei (b), and total number of metabolites identified (c). In total, 460 and 477 metabolites were putatively identified in $E$ coli and T. brucei extracts, respectively, using LC-MSbased metabolite profiling. 198 metabolites were determined as common chemical structures between both species

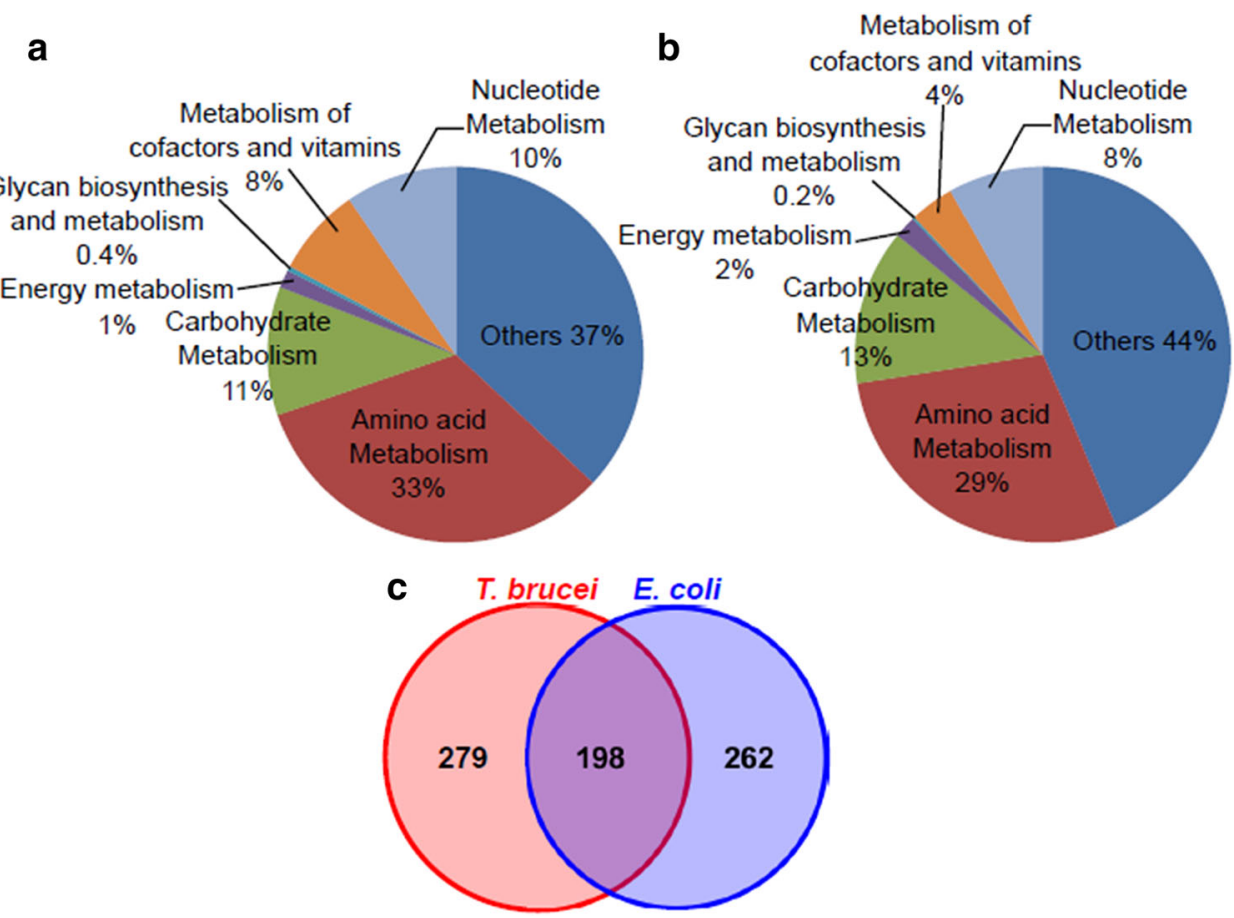

of amino acids were compared to measurements in Trypanosoma gambiense and T. brucei reported in Chappell et al. (1972) and Smith et al. (2009). As can be seen in Fig. 2, overall, our measurements are matched relatively well with Chappell et al.: alanine is by far the most abundant free amino acid in both media as well as in Chappell et al. representing $49 \%$ of the total free amino acid pools in HMI-9, $62 \%$ in CMM and $47 \%$ for Chappell et al. This is also the case in Trypanosoma cruzi (O'Daly et al. 1983) and Leishmania species (Simon et al. 1983; Vieira and Ioav Cabantchik 1995). Glutamate, serine and glycine represent more than $5 \%$ of the free amino acids pools in all three series of measurements. The main difference between our measurements and Chappell et al. is the fraction of proline and asparagine (5-9\% each in CMM and HMI-9 but low in Chappell et al.) and the fraction of arginine (about $8 \%$ in Chappell et al. about $2 \%$ in CMM and HMI-9). However, differences in growth conditions, species and environments might have caused these variations, and this could also explain differences between the measurements reported by Smith et al. and the results of other studies.

\subsection{Metabolic flux measurement using extracellular concentrations}

The measurement of extracellular metabolites excreted from cells into their growth media or consumed by the cells (Chong et al. 2009; Kaderbhai et al. 2003; Kim et al. 2010) can be connected to intracellular flux states (Mo et al.
2009). Therefore, quantitative analysis of extracellular metabolites in a growth medium can be employed for an understanding of cellular metabolism and the measurement of metabolic fluxes of many metabolites which can feed into quantitative computational models of metabolism.

Extracellular metabolites were sampled with $\mathrm{U}_{-}{ }^{13} \mathrm{C}-\mathrm{la}$ belled $E$. coli extract from the spent media at seven different time points $(0,7,24,31,48,51$ and $57 \mathrm{~h})$ and 32 metabolites were quantified by the IR-MS technique. It was confirmed in the previous study that no significant changes in any medium nutrient was observed in cell-free CMM incubated at $37{ }^{\circ} \mathrm{C}$ for $56 \mathrm{~h}$ (Creek et al. 2013). Extracellular concentrations from different time points are shown in Supplementary Table S4. Among these 32 metabolites, the concentrations of seven metabolites do not change over time in the medium or the variations are too small to allow flux quantification (see Fig. 3). It was previously shown that $T$. brucei lacks an arginase (Vincent et al. 2012), the enzyme that converts L-arginine to L-ornithine present notably in Leishmania (Roberts et al. 2004), and that $T$. bruce $i$ is capable of taking up L-ornithine from the medium (Vincent et al. 2012). Our results show that significant amounts of L-ornithine are not consumed in either CMM or HMI-9. However, assuming that cells require ornithine only to synthesise putrescine, spermidine and trypanothione, we can estimate the total concentration of ornithine required per cell as about $6.7 \mathrm{mM}$ for cells grown in CMM and $5.2 \mathrm{mM}$ in HMI-9 [using the concentrations of ornithine, putrescine and spermidine in Table 1 and the concentration of trypanothione $(160 \mu \mathrm{M})$ measured by 
Table 1 The concentrations of intracellular metabolites in $T$. brucei grown in HMI-9 and $\mathrm{CMM}$

\begin{tabular}{|c|c|c|}
\hline Name & Concetration in HMI-9 $(\mu \mathrm{M} \pm \mathrm{SEM})$ & Concetration in $\mathrm{CMM}(\mu \mathrm{M} \pm \mathrm{SEM})$ \\
\hline$N$-Acetylornithine & $0.2 \pm 0.1$ & $0.2 \pm 0.0$ \\
\hline cis-Aconitate & $0.3 \pm 0.0$ & $0.3 \pm 0.0$ \\
\hline Adenosine & $28.2 \pm 2.2$ & $12.2 \pm 1.3$ \\
\hline S-Adenosylmethionine & $38.5 \pm 2.9$ & $44.7 \pm 1.2$ \\
\hline L-Alanine & $21439.1 \pm 246.8$ & $32754.3 \pm 1334.0$ \\
\hline 4-Aminobutanoate & $19.4 \pm 1.8$ & $21.5 \pm 2.4$ \\
\hline L-Arginine & $777.8 \pm 28.9$ & $1065.8 \pm 111.9$ \\
\hline L-Asparagine & $2886.0 \pm 96.2$ & $2595.4 \pm 121.8$ \\
\hline L-Aspartate & $395.0 \pm 37.6$ & $609.2 \pm 80.2$ \\
\hline Citrate & $53.8 \pm 5.7$ & $57.7 \pm 4.0$ \\
\hline Fumarate & $347.2 \pm 44.4$ & $305.0 \pm 40.5$ \\
\hline L-Glutamate & $2769.4 \pm 130.8$ & $3502.7 \pm 396.3$ \\
\hline L-Glutamine & $2469.5 \pm 220.5$ & $1736.3 \pm 111.8$ \\
\hline Glycine & $3498.8 \pm 94.5$ & $2987.6 \pm 58.9$ \\
\hline L-Histidine & $245.5 \pm 8.1$ & $238.4 \pm 5.5$ \\
\hline (R)-2-Hydroxyglutarate & $128.6 \pm 4.0$ & $109.8 \pm 4.7$ \\
\hline Hypoxanthine & $325.5 \pm 31.5$ & $1.4 \pm 0.1$ \\
\hline L-Isoleucine & $299.2 \pm 25.1$ & $82.1 \pm 10.4$ \\
\hline L-Lysine & $346.2 \pm 40.5$ & $133.2 \pm 5.3$ \\
\hline (S)-Malate & $324.8 \pm 36.2$ & $287.2 \pm 40.6$ \\
\hline L-Methionine & $156.9 \pm 6.5$ & $112.1 \pm 5.6$ \\
\hline $5^{\prime}$-Methylthioadenosine & $2.1 \pm 0.2$ & $2.3 \pm 0.1$ \\
\hline Nicotinamide & $25.6 \pm 2.9$ & $14.5 \pm 0.3$ \\
\hline L-Ornithine & $407.3 \pm 25.4$ & $1072.8 \pm 58.5$ \\
\hline Orotate & $<0.3$ & $<0.3$ \\
\hline 2-Oxoglutarate & $845.9 \pm 35.1$ & $944.3 \pm 131.3$ \\
\hline Pantothenate & $6.7 \pm 0.5$ & $0.3 \pm 0.1$ \\
\hline L-Phenylalanine & $131.3 \pm 12.0$ & $48.2 \pm 3.1$ \\
\hline Phenylpyruvate & $92.5 \pm 3.8$ & $51.1 \pm 2.7$ \\
\hline 3-Phospho-D-glycerate & $2251.7 \pm 60.7$ & $1101.1 \pm 110.8$ \\
\hline Phosphoenolpyruvate & $52.6 \pm 4.0$ & $27.0 \pm 2.2$ \\
\hline L-Proline & $3835.8 \pm 277.2$ & $2688.7 \pm 177.5$ \\
\hline Putrescine & $1157.0 \pm 23.2$ & $1282.8 \pm 29.7$ \\
\hline Pyruvate & $7147.3 \pm 185.3$ & $7092.3 \pm 637.5$ \\
\hline L-Serine & $3066.4 \pm 59.6$ & $2931.0 \pm 91.2$ \\
\hline Spermidine & $3490.7 \pm 58.0$ & $4203.9 \pm 235.4$ \\
\hline Succinate & $2914.4 \pm 148.7$ & $2975.4 \pm 198.2$ \\
\hline L-Tryptophan & $30.9 \pm 8.7$ & $12.4 \pm 3.5$ \\
\hline L-Tyrosine & $331.5 \pm 21.6$ & $126.0 \pm 3.6$ \\
\hline UDP-Glucose & $915.3 \pm 35.6$ & $1126.1 \pm 46.5$ \\
\hline Uracil & $8.9 \pm 0.4$ & $6.5 \pm 0.6$ \\
\hline L-Valine & $504.6 \pm 30.2$ & $143.4 \pm 15.2$ \\
\hline Xanthine & $439.2 \pm 18.0$ & $111.6 \pm 38.5$ \\
\hline
\end{tabular}

(Henderson et al. 1987)]. Given a cell volume of $5.89 \mu \mathrm{l} /$ $10^{8}$ cells (Ariyanayagam et al. 2005), the total quantity of ornithine required per cell is $3.96 \times 10^{-10} \mu \mathrm{mol}$ in CMM and $3.07 \times 10^{-10} \mu \mathrm{mol}$ in HMI-9 and considering the cell density after $57 \mathrm{~h}$ of culture (about $4.6 \times 10^{6}$ cells $/ \mathrm{ml}$ in
CMM and $5.4 \times 10^{6}$ cells $/ \mathrm{ml}$ in HMI-9), the total concentration of ornithine in the medium would have decreased by $1.8 \mu \mathrm{M}\left(1.8 \times 10^{-3} \mu \mathrm{mol} / \mathrm{ml}\right)$ in $\mathrm{CMM}$ and $1.7 \mu \mathrm{M}$ in HMI-9 after $57 \mathrm{~h}$. This is clearly too small for us to see, given the variations of our measurement (see 
Fig. 2 Comparison of the percentage of each amino acid in total intracellular amino acid concentrations in T. brucei to measurements reported in Chappell et al. (1972) and Smith et al. (2009)
Fig. 3 Variations of concentrations of the metabolites measured in CMM (unless otherwise specified) for which neither consumption nor production can be established over $57 \mathrm{~h}$. The measurements where done in negative mode unless "+" is specified. Each type of symbol corresponds to a biological replicate of the time course
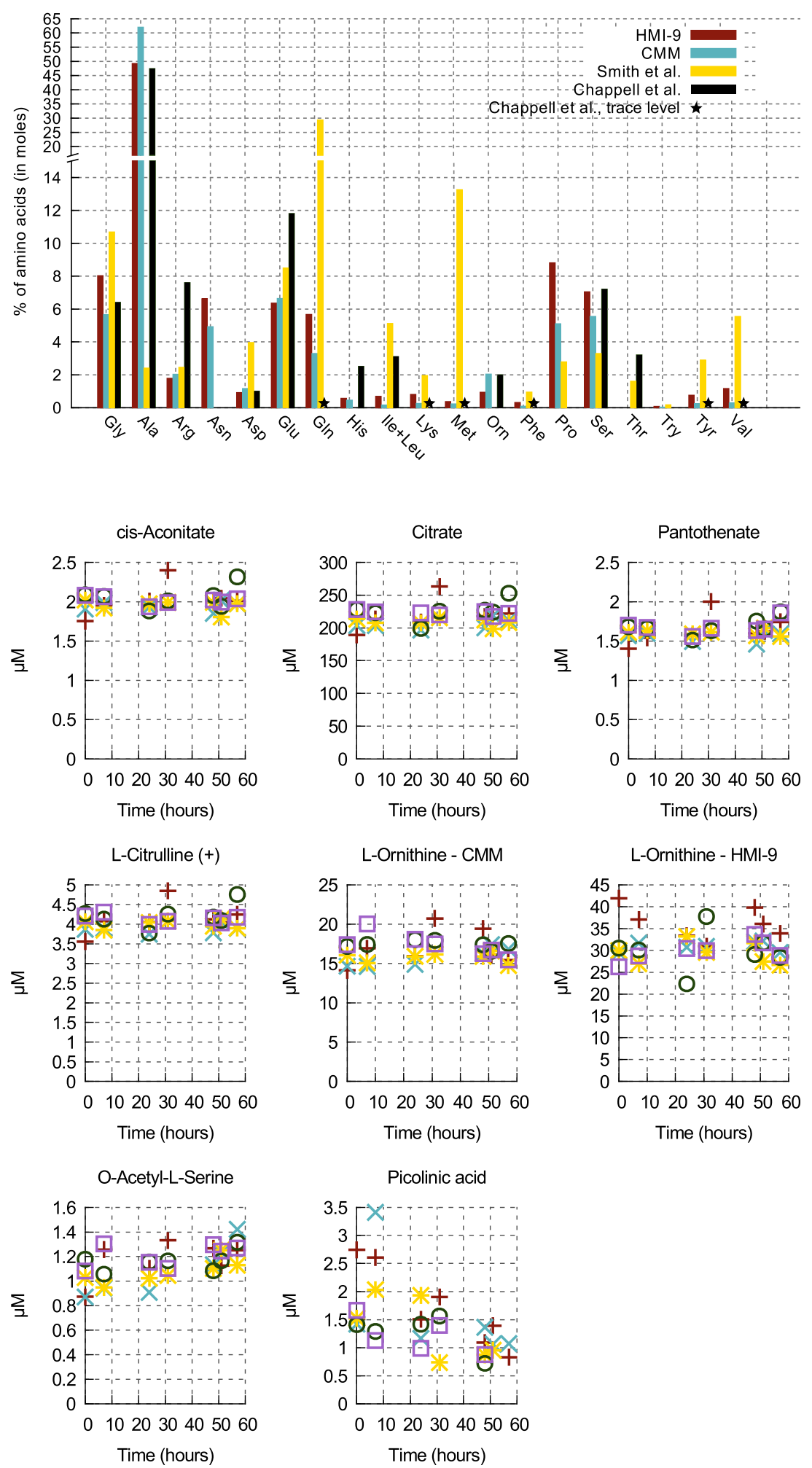

Fig. 3). This is also likely to be the case for pantothenate, which is required for coenzyme A biosynthesis.

For 19 metabolites, the time courses were not consistent with a constant production or consumption (see Fig. 4a, b).
These metabolites are either amino acids (Fig. 4a) or nucleosides/nucleotides (Fig. 4b; Supplementary Figure S6). All of these compounds showed a similar pattern: over the first $31 \mathrm{~h}$, while the cell number is still relatively 
low, the concentration of these metabolites increased; after $31 \mathrm{~h}$ they either decrease or continue to increase depending on the metabolite; in none of the cases do they show the exponential increase that would result from a constant production rate. It has been shown that $T$. brucei secretes numerous proteins, such as enzymes involved in nucleotide and protein hydrolysis (Geiger et al. 2010; Holzmuller et al. 2010; Troeberg et al. 1996). Therefore, the concentration increase in the first $31 \mathrm{~h}$ is most likely the result of amino acid and nucleotide released from proteins and nucleic acids respectively; with low cell density, the rate of production of these metabolites exceeds their consumption by the parasites. By $48 \mathrm{~h}$, as the cell density increases and depending on the metabolite and its concentration: (i) the metabolite is now consumed more than it is produced, hence its disappearance from the medium, or (ii) it is produced more than it is consumed and it appears to increase (slowly) over time. Some metabolites are also produced by the parasite and excreted, and hence continue to increase, albeit at a decreasing rate.

Finally, six metabolites appear to be constantly produced or consumed and thus show an exponential increase or decrease over time (see Fig. 5a), allowing quantification of their production or consumption (see Sect. 2) when cells
Fig. 4 Concentrations of amino acids (a) and nucleoside/ nucleotide (b) in CMM medium (negative mode unless otherwise specified) over $57 \mathrm{~h}$. Each type of symbol corresponds to a biological replicate of the time course a
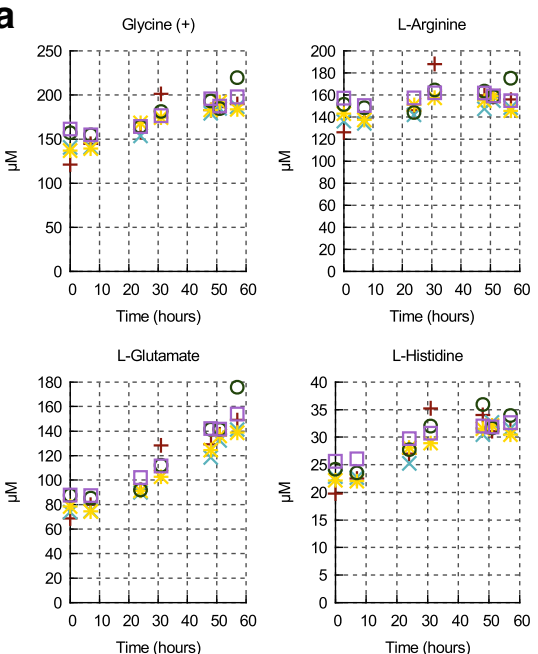

L-Methionine S-oxide (+)

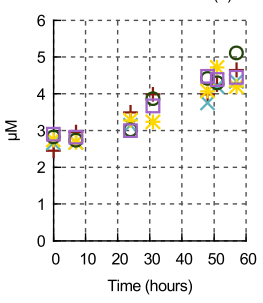

L-Serine (+)

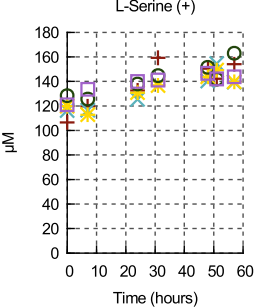

b

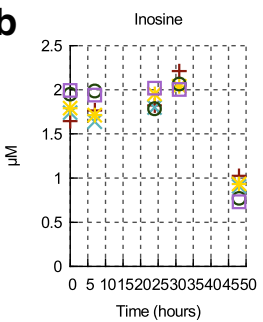

L-Phenylalanine $(+)$

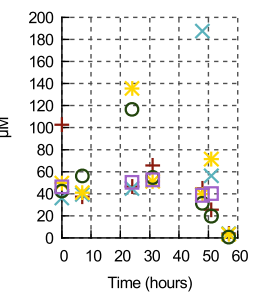

Time (hours)

L-Tryptophan

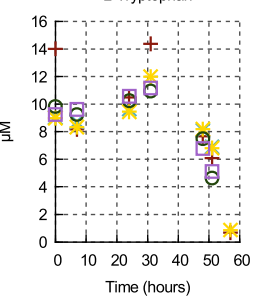

Xanthine

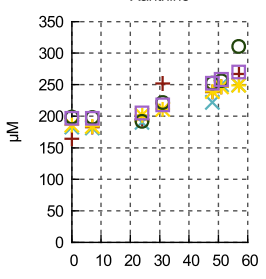

Time (hours)
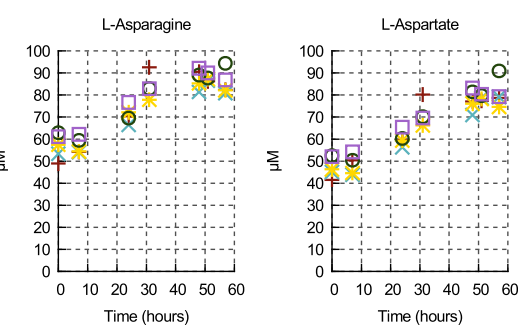

L-Lysine
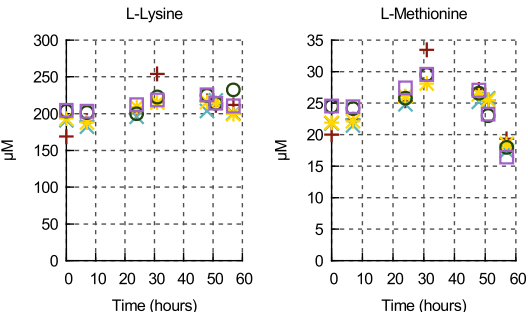

L-Proline

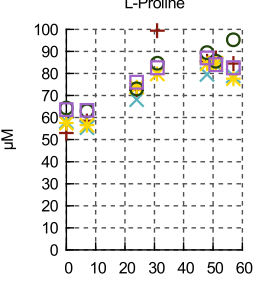

Time (hours)
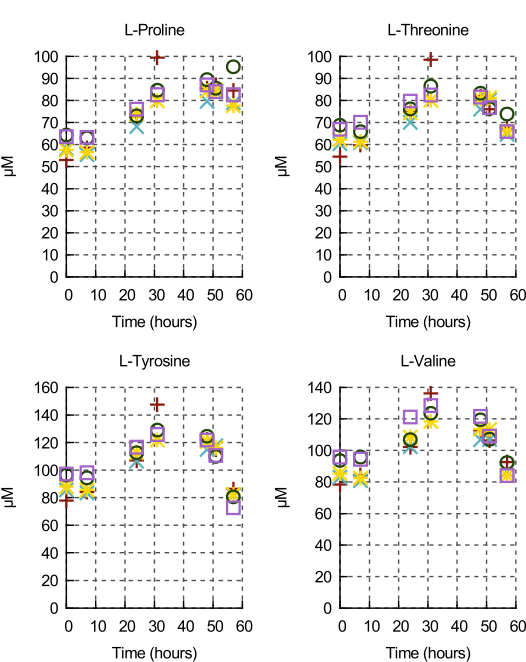

Time (hours)

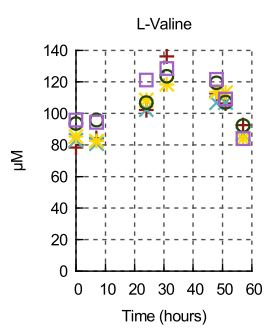

Hypoxanthine

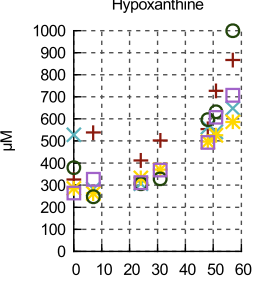

Time (hours) 
are grown in CMM and HMI-9 (see Fig. 5b). Glutamine is added to the medium both in CMM (1 mM) and HMI-9 ( $4 \mathrm{mM}$ ) and is consumed rapidly (compared to other amino acids); therefore, any small increase due to peptidase activity is not significant. Similarly, L-alanine is produced from pyruvate and excreted rapidly by the cells, hence any increase due to peptidase action in the medium is not significant and fluxes can be estimated. As expected, the pyruvate production flux is the highest flux measured. This flux is the same whether cells are grown in CMM or HMI-9 medium. The pyruvate flux we measured in HMI-9 is substantially higher than the flux reported by Haanstra et al. (2012). They measured about $370 \mathrm{nmol} /\left(\min 10^{8}\right.$ cells) (calculated from the glucose consumption flux and the pyruvate production to glucose consumption ratio) whilst we measured $680 \pm 47 \mathrm{nmol} /\left(\min 10^{8}\right.$ cells $)$ despite similar growth rates (see Supplementary Figure S7). A difference between experiments in which flux was measured here and the results of Haanstra et al. is the cell density at the beginning of the time-course, which might account for the measured differences.

In addition to pyruvate, L-alanine, 2-oxoglutarate, succinate and glycerate are constantly produced by the cells. The L-alanine produced has been shown to come primarily from pyruvate (Creek et al. 2015) via alanine aminotransferase (Ariyanayagam et al. 2005). Hence the glutamine consumed is probably used as an amino group donor, which would explain why in HMI-9 where the alanine production is higher, the glutamine consumption is also higher. Each glutamine can donate up to two amino groups
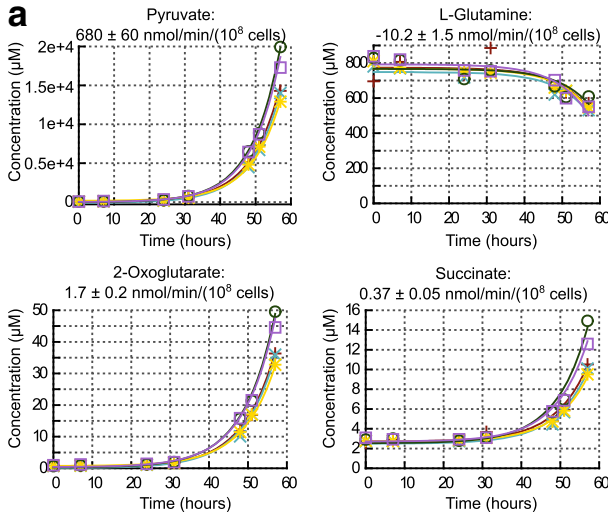

b
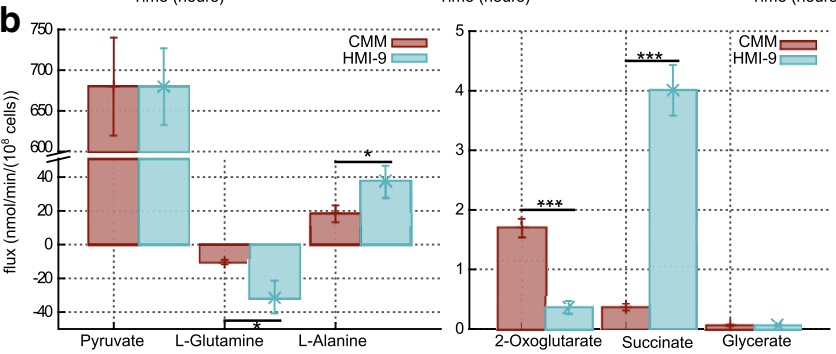
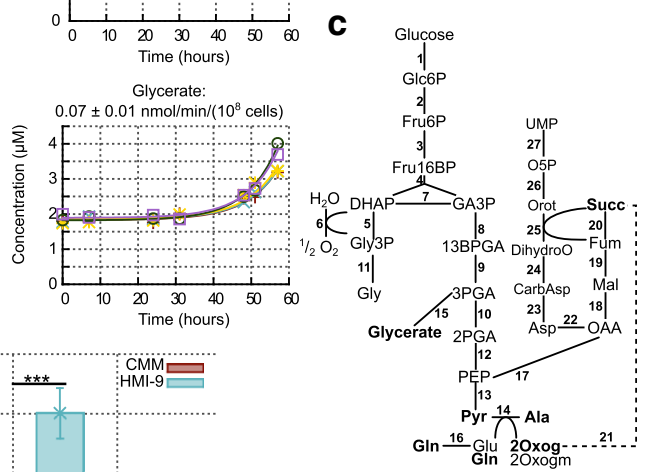

Fig. 5 a Time-courses and fitted fluxes of constantly consumed or produced metabolites in CMM medium (negative mode). b Fluxes of constantly consumed or produced metabolites in both CMM and HMI-9 medium. Each type of symbol corresponds to a biological replicate of the time course. $* p$ value $<0.05, * * * p$ value $<0.001$. c Simplified schematic of the part of bloodstream form $T$. brucei metabolism connected to the elements measured in (a), based on (Creek et al. 2015). Glc6P glucose 6-phosphate, Fru6P fructose 6-phosphate, Fru16BP fructose 1,6-bisphosphate, DHAP dihydroxyacetone phosphate, GA3P glyceraldehyde 3-phosphate, Gly3P glycerol 3-phosphate, Gly glycerol, 13BPGA 1,3bisphosphoglyceraldehyde, 3PGA 3-phosphoglyceraldehyde, 2PGA 2-phosphoglyceraldehyde, $P E P$ phosphoenolpyruvate, $P y r$ pyruvate, Ala L-alanine, Gln L-glutamine, Glu -glutamate, 2Oxog 2-oxoglutarate, 2Oxogm 2-oxoglutaramate, OAA oxaloacetate, Mal malate, Fum fumarate, Succ succinate, Asp L-aspartate, CarbAsp carbamoylphosphate, CarbAsp N-carbamoyl-L-aspartate, DihydroO dihydroorotate, Orot orotate, O5P orotate 5-phosphate. Reactions 1 hexokinase, 2 phosphoglucose isomerase, 3 phosphofructokinase, 4 aldolase,
5 glycerol 3-phosphate dehydrogenase, 6 glycerol 3-phosphate dehydrogenase + trypanosome alternative oxidase, 7 triosephosphate isomerase, 8 glyceraldehyde 3-phosphate dehydrogenase, 9 phosphoglycerate kinase, 10 phosphoglycerate mutase, 11 glycerol kinase, 12 enolase, 13 pyruvate kinase, 14 alanine aminotransferase and glutamine-pyruvate transaminase (Marciano et al. 2009), 15 the labelling pattern of glycerate in (Creek et al. 2015) shows that it is most likely produced from triose-phosphate from glycolysis. The exact reaction and enzyme is not known, 16 glutaminase, 17 phosphoenolpyruvate carboxykinase, 18 malate dehydrogenase, 19 fumarase, 20 succinate dehydrogenase, 21 possible pathway producing succinate from 2-oxoglutarate. Exact reactions to be determined in bloodstream form, 22 aspartate aminotransferase, 23 aspartate carbamoyltransferase, 24 dihydroorotase, 25 dihydroorotate dehydrogenase, 26 orotate phosphoribosyltransferase, 27 orotidine- $5^{\prime}$-phosphate decarboxylase; part of this pathway is localised in the glycosomes, part is in the cytosol and part remains to be determined 
and other amino acids can also be used as amino group donors, explaining why alanine production flux can exceed the glutamine consumption flux. The cells then excrete either glutamate (see Fig. 4a) or 2-oxoglutarate or possibly 2-oxoglutaramate (annotation of the latter based on exact mass only). Interestingly, the cells excrete more 2-oxoglutarate in CMM than in HMI-9 despite consuming less glutamine. Two hypotheses could explain such a discrepancy: (i) the cells excrete more glutamate or 2-oxoglutaramate in HMI-9, which would mean that in the presence of more glutamate (4 mM in HMI-9, $1 \mathrm{mM}$ in CMM) the cells only use one of the two amino groups available in glutamine, (ii) the cells could further metabolise 2-oxoglutarate more in HMI-9. The latter hypothesis is further strengthened by the tenfold higher rate of production of succinate in HMI-9 as compared to CMM. Indeed, the succinate produced has been shown to come only in part from glucose, the rest most probably coming from amino acids (Creek et al. 2015). The small amount of glycerate produced comes from glucose (Creek et al. 2015) and is similar in both media.

It is worth noting that our measurements of the fluxes of succinate and alanine production are similar to those recently measured by Mazet et al. using NMR (Mazet et al. 2013) $\left[1.2 \mathrm{nmol} /\left(\operatorname{min~} 10^{8}\right.\right.$ cells $)$ of succinate and $14 \mathrm{nmol} /$ (min $10^{8}$ cells) of alanine], although the pyruvate production flux that they measured $\left[130 \mathrm{nmol} /\left(\min 10^{8}\right.\right.$ cells $)$ is significantly lower than that measured here or in Haanstra et al. (2012], probably due to stressful culture conditions in Mazet et al. (2013) (5 h in phosphate-buffered saline solution with added glucose but no other nutrients).

\subsection{Metabolites detected in the medium that could not be absolutely quantified}

Supplementary Figure 6 contains the time courses of metabolites identified in the medium (exact mass and retention time matching with their standards) that could not be quantified, either because the metabolite is absent in E. coli or because the concentration is beyond the dynamic range of our calibration curve (either in the medium or in E. coli). Among them, 11 compounds (L-cysteine, phenylpyruvate, imidazole4-acetate, hydroxyglutarate, glucuronolactone, glucono-1,4lactone, nicotinic acid, thymine and orotate) seem to be constantly produced or consumed. L-cysteine is known to be consumed in large quantities and is essential to T. brucei (Creek et al. 2013; Duszenko et al. 1992). Phenylpyruvate is the keto-acid produced from L-phenylalanine. Similarly, imidazole 4-acetate might be a degradation product or fragment of imidazole 4-pyruvate, the keto-acid produced from Lhistidine and hydroxyglutarate is the reduced form of 2-oxoglutarate, the keto-acid produced from L-glutamate. Indeed, it has long been known that $T$. brucei excretes numerous of these keto-acids (Berger et al. 1996; Creek et al. 2013; Stibbs and Seed 1973, 1975) that are produced via promiscuous aminotransferases (Ariyanayagam et al. 2005; Marciano et al. 2008).

Nicotinic acid is consumed by the cells where it is used in the production of nicotinamide-based cofactors e.g., $\operatorname{NAD}(\mathrm{H})$ and $\mathrm{NADP}(\mathrm{H})$. While it cannot be excluded with certainty that the detected compound is actually isonicotinic acid, based on exact mass and retention time similarity to the nicotinic acid standard, it is here putatively identified as nicotinic acid, as isonicotinic acid is not involved in any pathways or reactions documented for trypanosomes, in contrast to nicotinate which is a part of the NAD salvage pathway.

Thymine and orotate can be produced via degradation of nucleotides. They might therefore be expected to be acquired from medium as we see after $31 \mathrm{~h}$ for other nucleotides. However, since trypanosomes are capable of synthesising thymine and orotate from glucose (Creek et al. 2015) there is no observed decrease in the medium. Indeed, for thymine no uptake could be observed (Gudin et al. 2006).

The origin of the increased concentration of carbohydrate lactones is assumed to be the oxidation of glucose in the medium over the time course.

\section{Conclusion}

We report a practical methodology using an isotope ratiobased mass spectrometry technique for the measurement of intra- and extra-cellular concentrations of 43 metabolites in T. brucei and its spent medium. This study also demonstrates that the technique is a useful tool for the estimation of metabolic fluxes of the metabolites, providing an accurate and unbiased method for the analysis of intracellular metabolism. The computed metabolic fluxes will now allow us to identify the most plausible metabolic network topology and drive the extension of the existing computational models to a more comprehensive representation of the metabolic capacity of the parasite (Achcar et al. 2014; Bakker et al. 2010). Importantly, this absolute quantitative technique can be easily transferred to other organisms that cannot be grown on glucose as the sole carbon source, including human cells.

Acknowledgments This work was funded by the BBSRC (BB/ I004599/1) and NWO (NWO 826.09.007) as part of the SysMO "SilicoTryp" project (http://www.sysmo.net/index.php?index=164) and partly by core funding from the Wellcome Trust to the Wellcome Trust Centre for Molecular Parasitology (085349). We are also grateful to Darren J. Creek for his advice on the experiments. 


\section{Compliance with Ethical Standards}

Conflict of interest The authors declare that they have no conflict of interest.

Informed consent Informed consent was obtained from all individual participants included in the study

Open Access This article is distributed under the terms of the Creative Commons Attribution 4.0 International License (http://creativecommons.org/licenses/by/4.0/), which permits unrestricted use, distribution, and reproduction in any medium, provided you give appropriate credit to the original author(s) and the source, provide a link to the Creative Commons license, and indicate if changes were made.

\section{References}

Achcar, F., Barrett, M. P., \& Breitling, R. (2013). Explicit consideration of topological and parameter uncertainty gives new insights into a well-established model of glycolysis. FEBS Journal, 280, 4640-4651.

Achcar, F., Kerkhoven, E. J., The SilicoTryp Consortium, Bakker, B. M., Barrett, M. P., \& Breitling, R. (2012). Dynamic modelling under uncertainty: The case of Trypanosoma brucei energy metabolism. PLoS Computational Biology, 8, e1002352.

Achcar, F., et al. (2014). Chapter three: The Silicon trypanosome-A test case of iterative model extension in systems biology. Advances in Microbial Physiology, 64, 115-143.

Albert, M.-A., et al. (2005). Experimental and in silico analyses of glycolytic flux control in bloodstream form Trypanosoma brucei. Journal of Biological Chemistry, 280, 28306-28315.

Annesley, T. (2003). Ion suppression in mass spectrometry. Clinical Chemistry, 49, 1041-1044.

Ariyanayagam, M. R., Oza, S. L., Guther, M. L. S., \& Fairlamb, A. H. (2005). Phenotypic analysis of trypanothione synthetase knockdown in the African trypanosome. Biochemical Journal, 391, $425-432$.

Bakker, B. M., Mensonides, F. I. C., Teusink, B., van Hoek, P., Michels, P. A. M., \& Westerhoff, H. V. (2000). Compartmentation protects trypanosomes from the dangerous design of glycolysis. PNAS, 97, 2087-2092.

Bakker, B. M., Michels, P. A. M., Opperdoes, F. R., \& Westerhoff, H. V. (1997). Glycolysis in bloodstream form Trypanosoma brucei can be understood in terms of the kinetics of the glycolytic enzymes. Journal of Biological Chemistry, 272, 3207-3215.

Bakker, B. M., et al. (2010). The silicon trypanosome. Parasitology, 137, 1333-1341.

Barrett, M. P., Boykin, D. W., Brun, R., \& Tidwell, R. R. (2007). Human African trypanosomiasis: Pharmacological re-engagement with a neglected disease. British Journal of Pharmacology, $152,1155-1171$

Bennett, B. D., Kimball, E. H., Gao, M., Osterhout, R., Van Dien, S. J., \& Rabinowitz, J. D. (2009). Absolute metabolite concentrations and implied enzyme active site occupancy in Escherichia coli. Nature Chemical Biology, 5, 593-599.

Berger, B. J., Dai, W. W., Wang, H., Stark, R. E., \& Cerami, A. (1996). Aromatic amino acid transamination and methionine recycling in trypanosomatids. Proceedings of the National Academy of Sciences, 93, 4126-4130.

Brun, R., Blum, J., Chappuis, F., \& Burri, C. (2010). Human African trypanosomiasis. Lancet, 375, 148-159.

Chappell, L. H., Southworth, G. C., \& Read, C. P. (1972). Shortinterval absorption and metabolism of some amino acids in Trypanosoma gambiense. Parasitology, 64, 374-387.
Chokkathukalam, A., Jankevics, A., Creek, D. J., Achcar, F., Barrett, M. P., \& Breitling, R. (2013). mzMatch-ISO: An R tool for the annotation and relative quantification of isotope-labelled mass spectrometry data. Bioinformatics (Oxford, England), 29, 281-283.

Chong, W. P. K., et al. (2009). Metabolomics profiling of extracellular metabolites in recombinant Chinese Hamster Ovary fedbatch culture. Rapid Communications in Mass Spectrometry, 23, 3763-3771.

Creek, D. J., Chokkathukalam, A., Jankevics, A., Burgess, K. E. V., Breitling, R., \& Barrett, M. P. (2012). Stable isotope-assisted metabolomics for network-wide metabolic pathway elucidation. Analytical Chemistry, 84, 8442-8447.

Creek, D. J., Jankevics, A., Breitling, R., Watson, D. G., Barrett, M. P., \& Burgess, K. E. V. (2011). Toward global metabolomics analysis with hydrophilic interaction liquid chromatography-mass spectrometry: Improved metabolite identification by retention time prediction. Analytical Chemistry, 83, 8703-8710.

Creek, D., Nijagal, B., Kim, D., Rojas, F., Matthews, K., \& Barrett, M. (2013). Metabolomics guides rational development of a simplified cell culture medium for drug screening against Trypanosoma brucei. Antimicrobial Agents and Chemotherapy, 57, 2768-2779.

Creek, D. J., et al. (2015). Probing the metabolic network in bloodstream-form Trypanosoma brucei using untargeted metabolomics with stable isotope labelled glucose. PLoS Pathogens, 11 , e1004689.

Delespaux, V., \& de Koning, H. P. (2007). Drugs and drug resistance in African trypanosomiasis. Drug Resistance Updates, 10, $30-50$.

Denery, J. R., Nunes, A. A. K., Hixon, M. S., Dickerson, T. J., \& Janda, K. D. (2010). Metabolomics-based discovery of diagnostic biomarkers for onchocerciasis. PLoS Neglected Tropical Diseases, 4, e834.

Duszenko, M., Mühlstädt, K., \& Broder, A. (1992). Cysteine is an essential growth factor for Trypanosoma brucei bloodstream forms. Molecular and Biochemical Parasitology, 50, 269-273.

Geiger, A., et al. (2010). Exocytosis and protein secretion in Trypanosoma. BMC Microbiology, 10, 20.

Gudin, S., et al. (2006). Trypanosoma brucei: A survey of pyrimidine transport activities. Experimental Parasitology, 114, 118-125.

Haanstra, J. R., et al. (2012). Proliferating bloodstream-form Trypanosoma brucei use a negligible part of consumed glucose for anabolic processes. International Journal for Parasitology, 42, 667-673.

Henderson, G. B., Fairlamb, A. H., \& Cerami, A. (1987). Trypanothione dependent peroxide metabolism in Crithidia fasciculata and Trypanosoma brucei. Molecular and Biochemical Parasitology, 24, 39-45.

Hirumi, H., Doyle, J. J., \& Hirumi, K. (1977). Cultivation of bloodstream Trypanosoma brucei. Bulletin of the World Health Organization, 55, 405-409.

Holzmuller, P., Grébaut, P., Cuny, G., \& Biron, D. G. (2010). Tsetse flies, trypanosomes, humans and animals: What is proteomics revealing about their crosstalks? Expert Review of Proteomics, 7, $113-126$.

Kaderbhai, N. N., Broadhurst, D. I., Ellis, D. I., Goodacre, R., \& Kell, D. B. (2003). Functional genomics via metabolic footprinting: Monitoring metabolite secretion by Escherichia coli tryptophan metabolism mutants using FT-IR and direct injection electrospray mass spectrometry. Comparative and Functional Genomics, 4, 376-391.

Kerkhoven, E. J., et al. (2013). Handling uncertainty in dynamic models: The pentose phosphate pathway in Trypanosoma brucei. PLoS Computational Biology, 9, e1003371. 
Kim, D.-H., et al. (2010). Combining metabolic fingerprinting and footprinting to understand the phenotypic response of HPV16 E6 expressing cervical carcinoma cells exposed to the HIV antiviral drug lopinavir. Analyst, 135, 1235-1244.

Marciano, D., Maugeri, D. A., Cazzulo, J. J., \& Nowicki, C. (2009). Functional characterization of stage-specific aminotransferases from trypanosomatids. Molecular and Biochemical Parasitology, 166, 172-182.

Marciano, D., et al. (2008). Biochemical characterization of stagespecific isoforms of aspartate aminotransferases from Trypanosoma cruzi and Trypanosoma brucei. Molecular and Biochemical Parasitology, 161, 12-20.

Mashego, M., et al. (2004). MIRACLE: Mass isotopomer ratio analysis of $\mathrm{U}_{-}{ }^{13} \mathrm{C}$-labeled extracts. A new method for accurate quantification of changes in concentrations of intracellular metabolites. Biotechnology and Bioengineering, 85, 620-628.

Mazet, M., et al. (2013). Revisiting the central metabolism of the bloodstream forms of Trypanosoma brucei: production of acetate in the mitochondrion is essential for parasite viability. PLoS Neglected Tropical Diseases, 7, e2587.

Mo, M., Palsson, B., \& Herrgard, M. (2009). Connecting extracellular metabolomic measurements to intracellular flux states in yeast. BMC Systems Biology, 3, 37.

O’Daly, J. A., Serrano, L. E., \& Rodríguez, M. B. (1983). Free aminoacid pool and proteolytic enzymes in Trypanosoma cruzi cultured in vitro. International Journal for Parasitology, 13, 433-440.

Rabinowitz, J. D., \& Kimball, E. (2007). Acidic acetonitrile for cellular metabolome extraction from Escherichia coli. Analytical Chemistry, 79, 6167-6173.

Roberts, S. C., Tancer, M. J., Polinsky, M. R., Gibson, K. M., Heby, O., \& Ullman, B. (2004). Arginase plays a pivotal role in polyamine precursor metabolism in Leishmania. Characterization of gene deletion mutants. The Journal of Biological Chemistry, 279, 23668-23678.

Scheltema, R. A., Jankevics, A., Jansen, R. C., Swertz, M. A., \& Breitling, R. (2011). PeakML/mzMatch: A file format, Java library, R library, and tool-chain for mass spectrometry data analysis. Analytical Chemistry, 83, 2786-2793.

Shi, G. (2003). Application of co-eluting structural analog internal standards for expanded linear dynamic range in liquid chromatography/electrospray mass spectrometry. Rapid Communications in Mass Spectrometry, 17, 202-206.

Simon, M. W., Jayasimhulu, K., \& Mukkada, A. J. (1983). The free amino acid pool in Leishmania tropica promastigotes. Molecular and Biochemical Parasitology, 9, 47-57.

Smith, T. K., et al. (2009). Blocking variant surface glycoprotein synthesis in Trypanosoma brucei triggers a general arrest in translation initiation. PLoS One, 4, e7532.

Stibbs, H. H., \& Seed, J. R. (1973). Chromatographic evidence for the synthesis of possible sleep-mediators in Trypanosoma brucei gambiense. Experientia, 29, 1563-1565.
Stibbs, H. H., \& Seed, J. R. (1975). Further studies on the metabolism of tryptophan in Trypanosoma brucei gambiense: Cofactors, inhibitors, and end-products. Experientia, 31, 274-278.

Sumner, L., Lei, Z., Nikolau, B., Saito, K., Roessner, U., \& Trengove, R. (2014). Proposed quantitative and alphanumeric metabolite identification metrics. Metabolomics, 10, 1047-1049.

Sumner, L. W., et al. (2007). Proposed minimum reporting standards for chemical analysis Chemical Analysis Working Group (CAWG) Metabolomics Standards Initiative (MSI). Metabolomics, 3, 211-221.

t'Kindt, R., et al. (2010). Towards an unbiased metabolic profiling of protozoan parasites: Optimisation of a Leishmania sampling protocol for HILIC-orbitrap analysis. Analytical and Bioanalytical Chemistry, 398, 2059-2069.

Tautenhahn, R., Bottcher, C., \& Neumann, S. (2008). Highly sensitive feature detection for high resolution LC/MS. BMC Bioinformatics, 9, 504.

Theodoridis, G. A., Gika, H. G., Want, E. J., \& Wilson, I. D. (2012). Liquid chromatography-mass spectrometry based global metabolite profiling: A review. Analytica Chimica Acta, 711, $7-16$.

Troeberg, L., Pike, R. N., Morty, R. E., Berry, R. K., Coetzer, T. H. T., \& Lonsdale-Eccles, J. D. (1996). Proteases from Trypanosoma brucei brucei. European Journal of Biochemistry, 238, 728-736.

van der Kloet, F., et al. (2012). Discovery of early-stage biomarkers for diabetic kidney disease using ms-based metabolomics (FinnDiane study). Metabolomics, 8, 109-119.

Vieira, L. L., \& Ioav Cabantchik, Z. (1995). Amino acid uptake and intracellular accumulation in Leishmania major promastigotes are largely determined by an $\mathrm{H}^{+}$-pump generated membrane potential. Molecular and Biochemical Parasitology, 75, 15-23.

Vincent, I., Creek, D., Burgess, K., Woods, D., Burchmore, R., \& Barrett, M. (2012). Untargeted metabolomics reveals a lack of synergy between nifurtimox and eflornithine against Trypanosoma brucei. PLoS Neglected Tropical Diseases, 6, e1618.

Vuckovic, D. (2012). Current trends and challenges in sample preparation for global metabolomics using liquid chromatography-mass spectrometry. Analytical and Bioanalytical Chemistry, 403, 1523-1548.

Winder, C. L., et al. (2008). Global metabolic profiling of Escherichia coli cultures: An evaluation of methods for quenching and extraction of intracellular metabolites. Analytical Chemistry, 80, 2939-2948.

$\mathrm{Wu}$, L., et al. (2005). Quantitative analysis of the microbial metabolome by isotope dilution mass spectrometry using uniformly ${ }^{13} \mathrm{C}$-labeled cell extracts as internal standards. Analytical Biochemistry, 336, 164-171.

$\mathrm{Xu}, \mathrm{Y}$., et al. (2010). Evaluation of accurate mass and relative isotopic abundance measurements in the LTQ-Orbitrap mass spectrometer for further metabolomics database building. Analytical Chemistry, 82, 5490-5501. 